\title{
BENEFICIOS DE ANTIOXIDANTES EN EL HÍGADO GRASO NO ALCOHÓLICO
}

\section{RESUMEN}

\author{
Gloria Zablah Kalil de Salem ${ }^{1}$ \\ José Alfonso Acevedo Santos ${ }^{2}$ \\ Recibido 07/10/16 \\ Aceptado: $23 / 12 / 16$
}

\begin{abstract}
El Hígado Graso No Alcohólico (HGNA) es una patología de creciente interés en la comunidad médica en general, por ser considerada el componente hepático del síndrome metabólico, asociado a dislipidemias, obesidad y diabetes mellitus. Teóricamente se ha considerado al estrés oxidativo como el factor determinante en su etiopatogenia, originado por una menor actividad antioxidante y el incremento de factores pro oxidante a partir de la hiperinsulinemia.
\end{abstract}

En este estudio se realiza el diagnóstico de hígado graso a través: de análisis clínicos, de laboratorio, ultrasonográficos, el NAFLD Score; además del método no tradicional: Sistema Cuántico Bioeléctrico (QRS), el cual presenta sensibilidad de un $85 \%$, que fue verificado con los hallazgos de laboratorio y ultrasonográficos. La investigación parte con un grupo de 28 pacientes que fueron diagnosticados como HGNA por el sistema cuántico (QRS), y de este grupo se seleccionaron cinco solo tres pacientes que cumplían todos los criterios de inclusión y exclusión de la muestra.

Los sujetos de estudio recibieron durante seis meses suplementación con cuatro cápsulas diarias que contenían 13 antioxidantes cada una. Como resultado se obtuvo la normalización de los parámetros clínicos, bioquímicos, ultrasonográficos y el sistema cuántico bioeléctrico de los tres sujetos de estudio sin modificaciones en la dieta ni en su actividad diaria.

PALABRAS CLAVE: Ácidos Grasos (AGs); Antioxidantes (AOX), Enfermedad de Hígado Graso No Alcohólica (EHGNA), Esteatosis Hepática No Alcohólica (EHNA); Hígado Graso No Alcohólico (HGNA); Hipertransaminasemia (AST, ALT); NALD, NASH; NAFLD, El Salvador.

\section{BENEFITS FROM ANTIOXIDANTS IN NON ALCOHOLIC FATTY LIVER}

\section{SUMMARY}

Non Alcoholic Fatty Liver (NAFL) is a disease of a great importance for medical community for being considered the hepatic factor in metabolic syndrom, associated to dyslipidemia, obesity and diabetes mellitus. Theorically, oxidative stress has been considered as the determinant factor in its ehiopatogeny, originated from a lower antioxidant activity and the rise of prooxidant factors starting from hyperinsulinemia.

In this research the diagnosis of fatty liver was made through: clinical and laboratory tests, ultrasound, NAFLD Score; besides the non traditional method Quantic Bioelectric System (QRS) which has an $85 \%$ sensibility that was verified through findings in laboratory and ultrasonographic tests. Research starts with a group of 28 patients who were diagnosed as NAFL

1 Departamento de Patología, Universidad Evangélica de El Salvador, g_i_salem@yahoo.com, número de filiación N-CONACYT 0831-110316

2 Departamento de Patología Universidad Evangélica de El Salvador, jose.alfonso.acevedo@gmail.com, número de filiación N-CONACYT 0861-300616 
through quantic system (QRS), and from this group there were 5 people selected and there were only 3 who met inclusion and exclusion criteria.

Subjects in research received supplements with 4 daily capsules for 6 months, and each one of them contained 13 antioxidants. As a result, a normal rate of clinical, biochemical and ultrasonographics parameters and bioelectrical quantic system from the subjects without diet or daily activities modifications.

Keywords: Fatty Acids(FA), Antioxidants (AO), Non Alcoholic Fatty Liver Disease(NAFLD), Non Alcoholic Hepatic Steatosis (NAHS), Non Alcoholic Fatty Liver (NAFL), Hypertransaminasemia (AST, ALT), NALD, NASH, NAFLD,

\section{INTRODUCCIÓN}

La esteatosis hepática simple no alcohólica (HGNA) es considerada actualmente un problema de salud, debido a los estilos de vida asociados a la alimentación inadecuada y subsistencia sedentaria. En el Salvador no se tienen cifras de la prevalencia e incidencia de hígado graso. Pero la Organización Mundial de la Salud (OMS) revela que existen al menos 400 millones de adultos obesos, de los cuales el $80 \%$ padecen de HGNA $^{1,2,3}$.

La esteatosis simple o acumulación intracelular de triglicéridos progresa a inflamación, fibrosis y cirrosis (Esteato Hepatitis No-Alcohólica, EHNA). La esteatosis simple es manifestación del síndrome metabólico cuya incidencia alcanza entre el 15 al $20 \%$ de la población con obesidad, de los cuales $3 \%$ presenta EHNA. En los diabéticos tipo II, la incidencia es del $50 \%$ de la población, de los cuales el 35\% presentan EHNA. En pacientes con obesidad mórbida, casi el $100 \%$ presenta EHNA ${ }^{4,5}$.

La obesidad, la diabetes Mellitus, el síndrome metabólico y dislipidemias constituyen patologías de alto riesgo relacionadas a HGNA y EHNA 4 .

El metabolismo hepático es aeróbico. Esto implica que existe una producción basal de Especies Reactivas de Oxigeno (EROS), que son consumidas normalmente por mecanismos antioxidante. El estrés oxidativo constituye un desequilibrio que favorece la oxidación, dañando moléculas esenciales, alterando la función biológica y sobrevida de la célula. Lo anterior se asocia a la activación de factores de transcripción redox sensibles, como el factor nuclear potenciador de las cadenas ligeras Kappa de las células B activadas (NF-Kb). Un complejo proteico que controla la trascripción del $A D N$ y el AP-1 o proteína activadora que controla la diferenciación, proliferación y apoptosis por las células de Kupffer y otras células no parenquimatosas ${ }^{5,6,7}$.

Según la Teoría del Primer y Segundo Golpe, el estrés oxidativo es el componente esencial que origina necrosis focal y fibrosis hepática, observándose menor actividad antioxidante que indica que la tasa de estrés oxidativo es el evento precoz y primordial en $\mathrm{EHNA}^{8}$.

Con base en lo anterior, el organismo humano necesita un flujo constante, abundante $y$ variado de antioxidantes diarios para combatir los radicales libres y las especies reactivos de oxígeno. $\mathrm{Y}$ así equilibrar el estrés oxidativo. Estudios actuales refieren que el organismo utiliza antioxidantes exógenos de la dieta como endógenos sintetizados en el organismo ${ }^{9,10}$.

Se han realizado estudios con agentes citoprotectores y antioxidantes como el Tocoferol (Vitamina E) en dosis variables de 300 a 1200 UI diarias al menos durante seis meses debido a que inhiben: Factor de Crecimiento de Transformación beta (TGF- $\beta$ ), las células de Ito, la peroxidación lipídica y suprimen citosinas proinflamatorias como Factor de Necrosis Tumoral alfa (TNF $\alpha$ ), obteniendo muy buenos resultados en HGNA ${ }^{10,11}$.

Es importante considerar que cada uno de los antioxidantes actúa en forma sinérgica e inter- 
dependiente. Además, la función de un antioxidante puede depender de la función apropiada de otro. Por lo anterior, se deduce que no es posible una terapia efectiva a través de un número reducido de antioxidantes. Actualmente no se ha podido resolver cuestionamientos tales como: ¿qué cantidades de antioxidantes necesita el paciente y cuáles serían los más aceptables? ${ }^{12}$.

- Mecanismo preventivo: a través de metaloenzima y oligoelementos.

- Mecanismo reparador: constituidos por enzimas que reparan/eliminan moléculas que han sido dañados por pro oxidantes. Ejemplos: el glutatión reductasa (GR), glutatión peroxidasa $(\mathrm{GPx})$ y el selenio.

- Mecanismo depurador: de radicales libres tales como las vitaminas C, E, carotenoides flavonoides, etc. Además de enzimas como: $\mathrm{Su}-$ peróxido Dismutasa (SDO) y Catalasa. Siendo los más utilizados en los estudios revisados las vitaminas $\mathrm{C}$ y $\mathrm{E}$, carotenoides y flavonoides ${ }^{13}$.

Considerando lo anterior, en este estudio se administraron a los pacientes suplementación por seis meses que incluyen antioxidantes que actúan en los tres niveles de defensa. El diagnóstico se realizó en base de herramientas de aplicación clínica: exploración física, antropométrica, exclusión de consumo de alcohol y de otras causas de hepatopatías; marcadores séricos e índices bioquímicos, elevación de transaminasas, menor de cuatro veces de su límite mayor; relación AST/ALT menor de uno; pruebas de imágenes y el QRS.

\section{METODOLOGÍA}

El estudio fue aprobado en septiembre del 2015 por el Comité de Ética de la Universidad Evangélica de El Salvador, según criterios internacionales de Helsinki.

El artículo muestra los resultados de una investigación de los beneficios de antioxidantes de dos femeninos y un masculino después que cada uno firmara el Consentimiento Informado que exige el comité de ética de la UEES. A cada paciente se le suministraron cuatro cápsulas diarias con 13 antioxidantes cada una.

Las características del grupo de estudio requeridas fueron: pacientes provenientes de consulta privada de residencia urbana, con obesidad central grado I, índice de masa corporal promedio de $30.4 \mathrm{Kg} /$ $m t s^{2}$, hipertransaminasemia crónica y con una edad promedio de 43 años.

\section{Criterios de Inclusión:}

- Pacientes con hipertransaminasemia crónica.

- $\quad$ Pacientes IMC mayor de $29 \mathrm{Kg} / \mathrm{mts}^{2}$.

- Pacientes con perímetro abdominal mayor de $80 \mathrm{~cm}$ en las mujeres y $102 \mathrm{~cm}$ en los hombres

- Pacientes que presentaban hígado graso a través del resultado QRS. Vale destacar que se utilizó el método QRS es un método diseñado para cuantificar el desbalance biomagnéticas en el organismo. A pesar que en la práctica actual no se le reconoce la validez científica que merece.

- Con ultrasonografía sugestiva a esteatosis hepática.

\section{Criterios de Exclusión:}

- Del estudio se excluyeron a los pacientes que consumían alcohol dos o más veces por semana, debido a que se ha considerado que la ingesta menor de 40 grs a la semana sería el límite para definir la esteatohepatitis no alcohólica ${ }^{14 .}$

- También se excluyó a los pacientes que padecían enfermedad hepática $u$ otra enfermedad específica como las enfermedades autoinmunes.

- Pacientes con positividad al antígeno de superficie del virus de la hepatitis B (Ag HBs) y anticuerpos positivos a Hepatitis C.

- Pacientes sin ultrasonografía hepática. 


\section{Tipo de Investigación}

El trabajo de investigación corresponde a la presentación de tres casos clínicos de pacientes con HGNA desde un enfoque prospectivo con una combinación de técnicas cualitativas y cuantitativas que tuvo como parámetros clínicos e instrumentos de análisis: exploración física, antropométrica, pruebas bioquímicas, ultrasonografía hepática y el Sistema Cuántico Bioeléctrico (QRS). Parámetros clínicos.

Existen varios métodos para diagnosticar el hígado graso, siendo el más confiable la biopsia hepática. Sin embargo, al ser de tipo invasivo, se prefiere el análisis clínico de laboratorio y ultrasonográficos, además de la puntuación del NAFLD score cuando existe esteatosis ${ }^{15,16,17}$.

\section{Hipertransaminasemia}

Las moderadas elevaciones de las transaminasas ALT o transaminasa pirúvica, obtenidas en dos ocasiones en un período mínimo de seis meses, asociadas a obesidad o sobrepeso incrementa la posibilidad de esteatosis hepática 55 .

\section{Ultrasonografía Hepática}

A tres pacientes se les realizó ultrasonografía hepática, siendo positiva a hígado graso. La ecografía hepática detecta el hígado graso arriba del 33\% del compromiso hepático. Recientemente un estudio chileno examinó la exactitud de la ultrasonografía hepática en 235 pacientes con enfermedad hepática que se sometieron a biopsia y mostró una sensibilidad de $64 \%$ y especificidad del $97 \%$, aumentando a $91 \%$ y $93 \%$, respectivamente, en pacientes con al menos $30 \%$ de esteatosis ${ }^{20,21,22}$

\section{Sistema Cuántico Bioeléctrico (QRS)}

En el estudio de HGNA se utiliza por primera vez un método no convencional, como: el Sistema Cuántico Bioeléctrico (QRS) diseñado para cuantificar las frecuencias y desbalances biomagnéticos en el organismo. El espectro de energía es cuantificable en el cuerpo humano y tiene su propia anatomía y por ello su propia escala de ondas electromagnéticas. El sensor que utiliza cuantifica los flujos magnéticos diminutos e imperceptibles fisiopatológicos propios de cada célula que, en esos momentos puede que presente o no cambios morfológicos reversibles o irreversibles a nivel en los tejidos hepáticos ${ }^{23}$.

Este método se considera alternativo por no estar incluido en los protocolos internacionales del manejo de Hígado Graso. Sin embargo, es un método económico y no invasivo con alta especificidad y sensibilidad del $85 \%$, de fácil ejecución y en corto período de tiempo. Además, este método recurre a la Serie de Fourier utilizado matemáticamente en la resonancia magnética nuclear, la ecocardiografía y otros campos como comunicaciones e ingeniería ${ }^{23 .}$

El QRS se basa en principios básicos del método científico: replicabilidad, racionalidad, principio teórico y principio de evidencia.

\section{NAFLD Score}

La puntuación de la fibrosis hígado graso no alcohólico es un modelo matemático y una herramienta no invasiva para la identificación de pacientes cuyo hígado graso no alcohólico ha progresado a la fibrosis hepática. Se basa en la información clínica disponible y los datos de laboratorio medidos de forma rutinaria. Este sistema de puntuación es recomendado por la Asociación Americana para el Estudio de las Enfermedades Hepáticas (AASLD), el Colegio Americano de Gastroenterología (ACG) y la Asociación Americana de Gastroenterología (AGA). Según el NAFLD Score los pacientes con una puntuación de fibrosis alta ( 3 ó 4 ) pueden necesitar estudios adicionales como la biopsia hepática o la elastografía no disponible en El Salvador. Dicho modelo también identifica a pacientes con bajo riesgo ( 1 y 2$)$ de presentar fibrosis los cuales pueden seguidos a intervalos periódicos ${ }^{24,25,26}$.

En línea se encuentra una calculadora de la puntuación de fibrosis que incluye: edad, IMC, glucemia/diabetes, AST, ALT, plaquetas $\left(10^{9} / \mathrm{L}\right)$ y albumina $(g r / L)$ sérica. 
La puntación de fibrosis o Score de fibrosis de EHNA es una herramienta clínica útil para identificar pacientes con esteato hepatitis (ENHA), con una alta probabilidad de presentar fibrosis o cirrosis. Un score menor de -1.455 excluye la probabilidad de fibrosis, una puntación entre -1.455 y 0.675 se considera indeterminada. Sin embargo, una puntación mayor de 0.675 se considera con alta probabilidad de fibrosis ${ }^{26}$.

Angulo et al consideran que la fibrosis en la esteatohepatitis (EHNA) comienza en la zona 3 del lobulillo hepático (región central o perivenular), adquiriendo un patrón peri celular y perisinusoidal. La fibrosis puede progresar hasta formar puentes porto-portales o veno-portales alterando la arquitectura del lobulillo por la formación de puentes hasta configurar la cirrosis que es la formación de nódulos regenerativos sin la presencia de la vena central ${ }^{26,27}$.

El estadio de fibrosis hepática de Brunt-Kleiner (Figura 1 y Tabla 1) que muestran varias características histopatológicas que en forma progresiva representan el curso de fibrosis peri celular hasta la formación de puentes o septos de tejido conectivo fibroso evidenciado con tinción especial de Tricromico de Masson, finalizando en la formación de nódulos regenerativos ${ }^{2829,30}$.

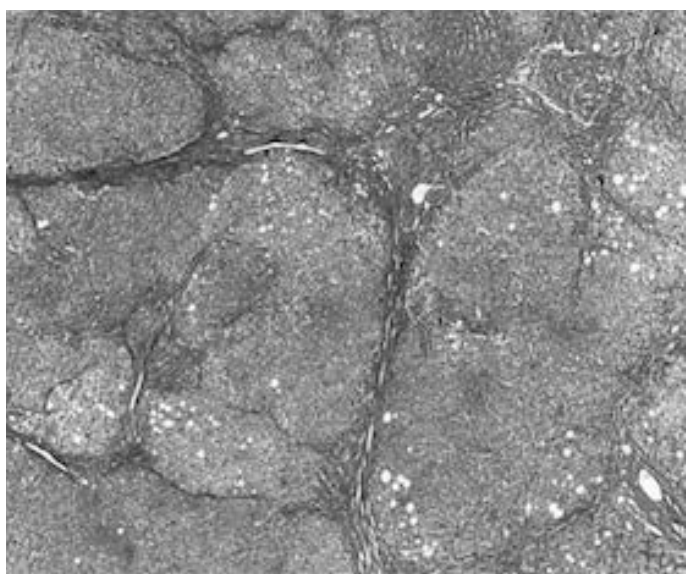

Figura 1. Estadios de fibrosis hepática Brunt-Kleiner. La imagen muestra fibrosis perisinusoidal, portal, peri portal y septas fibrosas en puentes, en una paciente de 62 años con cirrosis (estadio 4). Archivo Universidad Evangélica de El Salvador, en proceso de clasificación ${ }^{31}$.
La Tabla 1 muestra cómo la fibrosis progresa en estadios. En este estudio los sujetos se encontraban en estadio 0 se considera que si los pacientes se encuentran en estadios 1 y 2 pueden recibir antioxidantes.

Tabla 1. Estadio de fibrosis hepática Brunt-Kleiner.

\begin{tabular}{|l|c|}
\hline \multicolumn{1}{|c|}{$\begin{array}{c}\text { Estadios de } \\
\text { fibrosis }\end{array}$} \\
\hline $\begin{array}{l}\text { Ausencia de fibrosis } \\
\text { Fibrosis perisinusoidal /Peri } \\
\text { celular (zona 3) }\end{array}$ & 1 \\
$\begin{array}{l}\text { Fibrosis perisinusoidal /Peri } \\
\text { celular (zona 3) y portal /peri } \\
\text { portal }\end{array}$ & 2 \\
$\begin{array}{l}\text { Septal o puentes de fibrosis } \\
\text { Cirrosis }\end{array}$ & 3 \\
\hline
\end{tabular}

Fuente: elaboración propia.

\section{Tratamiento del grupo de estudio}

Los controles se realizaron mensualmente con el QRS durante los cuales se les proporciona a los pacientes suplementos antioxidantes por 30 días durante seis meses. A su vez, se les recomendó que no realizaran dietas hipocalóricas o mayor actividad física que la rutinaria. Además, se les proporcionó un suplemento que incluía antioxidantes que refuerza su mecanismo de prevención de formación de radicales libres, antioxidantes que promueven mecanismo reparador y antioxidantes secuestradores de radicales libres. Entre los antecedentes no existen estudios previos de terapia antioxidante realizados en esta forma, Pues se hizo un estudio de cada antioxidante de la fórmula y se clasificaron según los mecanismos antioxidantes ya anteriormente descritos.

- Dosis: 2 cápsulas.

- Periodicidad: cada 12 horas.

- Cantidad por dosis 2 cápsulas: Vitamina C $42.5 \mathrm{mg} 71 \%$ (Como ascorbato de calcio USP) Selenio (Como Selenometionina) $12.5 \mathrm{mcg} \quad 18 \%$ Riboceina D-Ribosa-LCisteína $125 \mathrm{mg}$ Fórmula exclusiva $580 \mathrm{mg}$ 
Cordyceps, Curcumina, Resveratrol, Acido Alpha Lipoico, Extracto de Semilla de Uva, Quercetina, Extracto de Cardo Mariano (Silybum Marianum), Extracto de Brócoli, Extracto de Aloe, BioPerene. Formulación de Cellgevety ${ }^{\oplus}$

\section{Antioxidantes administrados (Tabla 2):}

\section{Antioxidantes Secuestradores}

- La vitamina C junto con el Glutatión (GSH) juegan un rol importante en la destrucción de los intermediarios EROs. El GSH es un tripéptido, formado por los siguientes aminoácidos: ácido glutámico, glicina, cisteína. Este último es un aminoácido limitante para su síntesis, que a diferencia de los otros dos, no se recupera y su concentración intracelular es muy baja. Entre las principales funciones del GSH es mantener el balance redox en la célula y protegerla del stress oxidativo, nitrosativo y de los reactivos electrofílicos. Actúa como coenzima y participa en los procesos de destoxificacion y otros ${ }^{33,34,35}$.

- $\quad$ El Cordyceps sinensis (Ophiocorycep sinensis) es un hongo que se encuentra en los altiplanos del Tíbet, China y en el Himalaya. Uno de los componentes bioactivos más importantes del Cordyceps es la cordicepina. Posee un efecto modulador inmunológico y antiinflamatorio que mejora el estado energético de la célula y elimina los radicales libres de oxígeno $^{36}$.

- Resveratrol y Quercetina. Entre los mejores flavonoides investigados son el resveratrol y la quercetina con propiedades antioxidantes más potentes que el alfa tocoferol ${ }^{37}$.

- Curcumina. La Cúrcuma longa L es una planta de origen asiático y la curcumina es su ingrediente activo. Un compuesto fenólico que tiene un potente antioxidante sobre los ácidos grasos poli insaturados y en homogenados de órganos animales in vitro. Estas sustancias unidas a micelas de fosfatidilcolina inhibe la dioxigenación de ácidos grasos inducida por la lipooxigenasa. Se ha demostrado también que el metabolito más frecuente de Curcumina, la tetrahidrocurcumina, también inhibe la peroxidacion lipídica en micro somas hepáticos y en la membrana de los eritrocitos. La Curcumina es capaz de retirar los EROs responsables de la per oxidación lipídica y de eliminar, principalmente: el radical hidroxilo, el radical superóxido, el oxígeno singlete, el dióxido de nitrógeno y el óxido nítrico $38,39,40,41,42,4,44,45,46,47,48$.

- Extracto de semilla de uva. El extracto seco de semilla de uva contiene compuestos polifenólicos (catequinas, proantocianidinas) con actividad antioxidante, captadores de radicales libres mejores que la vitamina $\mathrm{C} \mathrm{y}$ E aisladas o en conjunto ${ }^{49}$.

\section{Antioxidantes Reparadores}

- Selenio. Los primeros estudios con selenio en seres vivos fue en la década de los $50 \mathrm{del}$ siglo 20 por Schawarz y Foltz. Los estudios consistían en la administración de selenio en ratas con necrosis hepática por déficit de vitamina E, demostrando que el selenio tiene una función antioxidante debido a la reducción de la necrosis hepática en las ratas. Los efectos beneficiosos del selenio están relacionados con el contenido de selenoproteínas o enzimas dependientes de selenio en el organismo. Todas ellas presentan propiedades antioxidantes y anti cancerígenas. El déficit de selenio provoca que las selenoproteinas y enzimas dependientes de selenio afecten a órganos como: hígado, músculo esquelético y el corazón, que presentan lesiones ante niveles carenciales. Además, niveles adecuados de selenio aumentan la absorción de vitaminas A, C, E y disminuyen la absorción de arsénico, cadmio y mercurio ${ }^{50,51,52}$.

- Acido Alfa Lipóico. También conocido como ácido tioctico o ácido lipoico se sintetiza a partir del ácido octanoico y se encuentra en todas las células. Se considera un antioxidante universal, siendo un potente 
antioxidante actuando en medios acuosos como lípidos. Posee 3 formas o niveles de acción:

$\diamond \quad$ Acción directa (secuestra radicales libres)

$\diamond \quad$ Efecto indirecto (es capaz de reciclar otros antioxidantes como la vitamina E, glutatión, coenzima Q y Vitamina C.

$\diamond \quad$ Mediante el incremento de síntesis celular de glutatión al incrementar la captación de cisteína y la expresión de la enzima limitante de su síntesis (gamma-glutamilcisteina ligasa) Entre sus múltiples beneficios está el de mejorar la capacidad de destoxicacion del hígado eliminando toxinas a través de glutatión durante la fase II de destoxificacion hepática ${ }^{53}$.

- Extracto de Cardo Mariano (Silybum Marianum). Los extractos del cardo mariano, Silybum marianum (L). Silimarina es el nombre colectivo de los flavonolignans (silybin o silibinina, silydianin, silycristin) extraído del cardo mariano. Se ha mostrado que estos extractos protegen a los animales contra diversas hepatotoxinas incluido el acetaminofén, la radiación y la sobrecarga de hierro. Las acciones hepatoprotector del cardo mariano pueden incluir la inhibición de la formación de peróxidos lipídicos, la eliminación de los radicales libres. Además, puede reducir la fibrogénesis hepática ${ }^{54}$.

- El extracto de semilla de brócoli (sulforafano glucosinolato) actúa como un catalizador para impulsar la actividad antioxidante hasta por tres días. Protege contra los electrófilos que dañan el ADN y ayuda a neutralizar las toxinas y células cancerígenas que son eliminadas de las células. También ayuda a elevar y reponer los niveles de glutatión. Induce a las enzimas usadas en la vía de desintoxicación de Fase II ${ }^{55}$.

\section{Antioxidantes Preventivos}

Aloe vera, tambien conocida como sábila, aloe de Barbados o aloe de Curazao. Contiene vitaminas del tipo A, C, B1, B2, B3, B6, B12 y ácido fólico. Además contiene calcio, zinc, cromo, selenio, hierro, cobre y magnesio ${ }^{56}$.

Bioperine (Pimienta negra). El ingrediente básico es un fitoquímico, la piperina, que aumenta el ritmo del metabolismo y por lo tanto quema calorias y conduce a la pérdida de peso. Aumenta la capacidad para absorber otros nutrientes como vitamina B y el Beta-caroteno ${ }^{57 .}$

Tabla 2. Clasificación de antioxidantes por mecanismos.

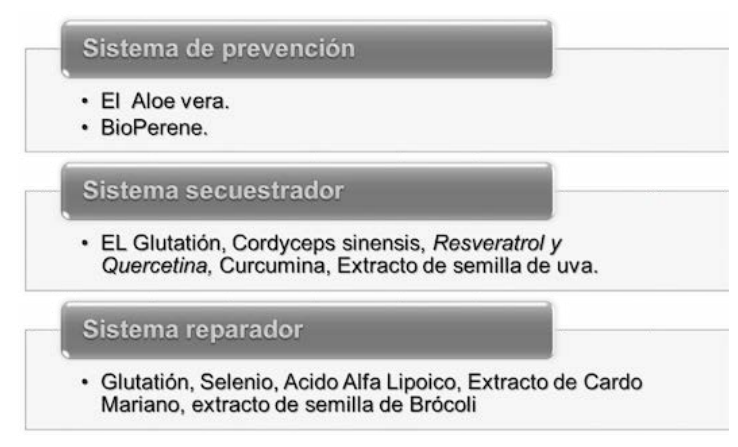

Fuente: elaboración propia con datos obtenidos de la Terapia Antioxidante y la formulación de Cellgevity ${ }^{\oplus}$

\section{RESULTADOS}

Al finalizar el tratamiento de seis meses se repitieron los análisis de laboratorio y ultrasonografía hepática con la finalidad de comprobar la ausencia de hígado graso, correlacionándolos con el QRS. Vale destacar que a los pacientes no se les intervino con dieta ni ejercicio.

Los tres pacientes presentaron: IMC mayor de 29 $\mathrm{Kg} / \mathrm{mt}^{2}$, clasificados como obesidad grado uno.

Paciente: CA001.

$\checkmark$ Paciente femenina de 33 años con IMC 29 y obesidad central, perímetro abdominal de: $91 \mathrm{cms}$. Los análisis de laboratorio mostraron: hipertransaminasemia en forma persistente de: ALT: $65 \mathrm{UI} / L, \mathrm{AST}=$ 52 UI/L. Relación AST/ALT: $0.80 \leq 1$. NAFLD Score: ausencia de fibrosis: $\mathrm{F} 0=-1.641$. Cuyos parámetros clínicos 
de laboratorio fueron: AST de $52 \mathrm{UI} / \mathrm{L}$; ALT $65 \mathrm{UI} / \mathrm{L}$; albumina de $41 \mathrm{~g} / \mathrm{L}$; glucosa de $87 \mathrm{mg} / \mathrm{dl}$; plaquetas 153000 . Ultrasonografía hepática: esteatosis moderada. Sistema QRS evidenció hígado graso de 0.69, considerada esteatosis $2+$.

$\checkmark \quad$ Resultado pos tratamiento: IMC: 28; perímetro abdominal $80 \mathrm{~cm}$; normalización de las transaminasas: ALT $33 \mathrm{UI} / L$; AST 23 UI/L. Ultrasonografía hepática: normal. Sistema Quantum Bioeléctrico: normalización de sus niveles de frecuencia diamagnética de HGNA 0.35 (Tabla 3).

Tabla 3. Tabla resumen caso CA001 de 33 años.

\begin{tabular}{|l|l|l|}
\hline \multicolumn{1}{|c|}{ CA001 } & \multicolumn{1}{c|}{$\begin{array}{c}\text { Pre } \\
\text { tratamiento }\end{array}$} & $\begin{array}{c}\text { Pos } \\
\text { tratamiento }\end{array}$ \\
\hline IMC & 29 & 28 \\
Perímetro abdominal & $92 \mathrm{cms}$ & $80 \mathrm{cms}$ \\
AST & 52 & 23 \\
ALT & 65 & 33 \\
Relación AST/ALT & $0.80 \leq 1$ & N/A \\
Albúmina & $41 \mathrm{~g} / \mathrm{L}$ & N/A \\
Plaquetas & 153000 & N/A \\
Glucosa & $87 \mathrm{mg} / \mathrm{Dl}$ & N/A \\
AgHbs Anti-HCV & Negativo & N/A \\
Ultrasonografía & Esteatosis & Negativo / \\
hepática & moderada & Normal \\
QRS & $0.692+$ & 0.33 (Negativo) \\
\hline
\end{tabular}

Fuente: elaboración propia.

Paciente: PP002

$\diamond$ Paciente masculino de 45 años con IMC32 y obesidad central perímetro abdominal $110 \mathrm{cms}$. Análisis de laboratorio mostró al final de los 6 meses: hipertransaminasemia persistente con: ALT: 76 UI/L, AST= 66 UI/. Relación AST/ALT: $0.86 \leq 1.46$. NAFLD Score. Puntuación indeterminada: F0: -1.012; albumina de $40 \mathrm{~g} / \mathrm{L}$; glucosa de $96 \mathrm{mg} /$ $d l$; plaquetas 197000 . Ultrasonografía hepática: esteatosis moderada. Sistema QRS: 0.72 considerada esteatosis $3+$. $\diamond$ Resultado pos tratamiento: IMC 31, perímetro abdominal de $97 \mathrm{~cm}$, normalización de las transaminasas: ALT: 33 UI/L; AST: 28 UI/L. Ultrasonografía Hepática: esteatosis leve difusa. Sistema QRS 0.33, normalización al quinto mes de tratamiento (Tabla 4).

Tabla 4. Tabla resumen Paciente PP002 de 45 años.

\begin{tabular}{|l|l|l|}
\hline PP002 & Inicio & $\begin{array}{l}\text { Pos } \\
\text { tratamiento }\end{array}$ \\
\hline IMC & 32 & 31 \\
Perímetro abdominal & $110 \mathrm{cms}$ & $97 \mathrm{cms}$ \\
AST & 66 & 28 \\
ALT & 76 & 33 \\
Relación AST/ALT & $0.86 \leq 1$ & N/A \\
Albúmina & $40 \mathrm{~g} / \mathrm{L}$ & N/A \\
Plaquetas & 197000 & N/A \\
Glucosa & $96 m g / D l$ & N/A \\
AgHbs Anti HCV & Negativo & N/A \\
Ultrasonografía hepática & Esteatosis & Esteatosis leve \\
& moderada & \\
QRS & $0.723+$ & 0.33 (Negativo) \\
\hline
\end{tabular}

Fuente: elaboración propia.

1. Paciente VH003.

$\diamond \quad$ Paciente femenina 54 años, IMC 30, obesidad central, perímetro abdominal: $95 \mathrm{cms}$; hipertransaminasemia de: ALT: $65 \mathrm{UI} / L, \mathrm{AST}=57 \mathrm{UI} /$; relación AST/ALT: $0.87 \leq 1$. NAFLD score: $F 1=-$ 1.927 Ausencia de fibrosis; albumina $41 \mathrm{mg} / \mathrm{dl}$; glucosa $106 \mathrm{mg} / \mathrm{dl}$; plaquetas 167000. Ultrasonografía hepática: esteatosis moderada. Sistema QRS: 0.71, considerada esteatosis $3+$.

$\diamond \quad$ Resultado pos tratamiento: IMC 30; perímetro abdominal $85 \mathrm{cms}$, normalización de las transaminasas: ALT: $31 \mathrm{UI} / L$; AST: 29 UI/L. Ultrasonografía hepática: normal. Sistema QRS: 0.34 normalización en el sexto mes (Tabla 5). 
Tabla 5. Tabla resumen paciente VH003 de 54 años.

\begin{tabular}{|l|l|l|}
\hline VH003 & Inicio & Pos tratamiento \\
\hline IMC & 30 & 30 \\
Perímetro abdominal & $95 \mathrm{cms}$ & $85 \mathrm{cms}$ \\
AST & 57 & 29 \\
ALT & 65 & 31 \\
Relación AST/ALT & $0.87 \leq 1$ & N/A \\
Albúmina & $41 \mathrm{~g} / \mathrm{L}$ & N/A \\
Plaquetas & 167000 & N/A \\
Glucosa & $106 \mathrm{mg} / \mathrm{Dl}$ & N/A \\
AgHbs AntiHCV & Negativo & N/A \\
Ultrasonografía & Esteatosis & Normal \\
hepática & moderada & \\
& & \\
QRS & $0.713+$ & 1.34 (negativo) \\
\hline
\end{tabular}

Fuente: elaboración propia.

Tabla 6. Esteatosis hepática. Sistema Cuántico Bioeléctrico (QRS). Medición mensual por 6 meses.

\begin{tabular}{|cccccccc|}
\hline $\begin{array}{c}\text { Código/ } \\
\text { paciente }\end{array}$ & $\begin{array}{c}\text { Rango } \\
\text { normal }\end{array}$ & $\begin{array}{c}1^{\circ} \\
\text { (inicial) }\end{array}$ & $2^{\circ}$ & $3^{\circ}$ & $4^{\circ}$ & $5^{\circ}$ & $\begin{array}{c}6^{\circ} \\
\text { (final) }\end{array}$ \\
\hline CA001 & $0.10-0.42$ & 0.69 & 0.67 & 0.61 & 0.53 & 0.44 & 0.35 \\
PP002 & $0.10-0.42$ & 0.72 & 0.69 & 0.61 & 0.53 & 0.41 & 0.39 \\
VH003 & $0.10-0.42$ & 0.71 & 0.71 & 0.63 & 0.57 & 0.43 & 0.34 \\
\hline
\end{tabular}

Fuente: elaboración propia.

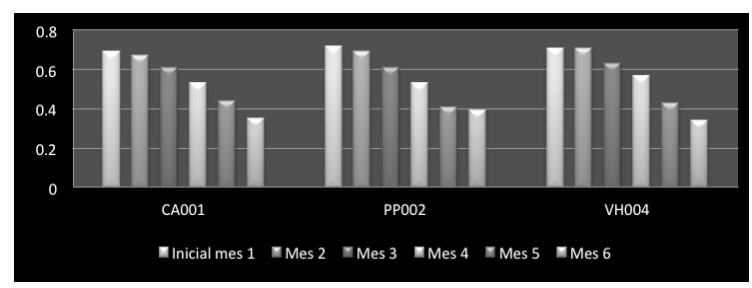

Figura 2. Sistema Cuántico Bioeléctrico. Elaboración propia de la medición mensual.

\section{ANÁLISIS Y DISCUSIÓN}

El organismo humano está en permanente equilibrio de su estado redox, necesitando la mayor cantidad de antioxidantes posibles (obtenidos generalmente con la dieta), para contrarrestar las moléculas pro oxidante. Además, posee sistema antioxidantes no enzimáticos y enzimáticos. Entre los primeros se tienen: el glutatión, ácido Lipóico, las ubiquinonas, bioflavonoides, vitamina E, C, B, coenzima Q, N-acetil cisteína, Resveratrol, selenio, etc. La dieta hipercalórico es un factor importante en la producción de EROS, que promueve la enfermedad hepática grasa no alcohólica, una de las causas más comunes de enfermedad hepática crónica en todo el mundo ${ }^{58}$.

Normalmente los ácidos grasos (AGs) son esenciales para el metabolismo hepático; sin embargo, en patologías como la obesidad, se altera el metabolismo intermediario hepático debido a la gran afluencia de hidratos de carbono y lípidos.

Los elevadosniveles de insulina(hiperinsulinemia) no son capaces de suprimir el flujo de $A G s$, el cual implica resistencia periférica de la insulina. El incremento del flujo de AGs circulantes es un factor importante en la patogénesis de HGNA. Tanto la hiperglucemia como la hiperinsulinemia favorecen la síntesis de la formación de triacilgliceridos TAGS, inhibiendo al $\beta$ - oxidación de $A G$, lo cual finaliza con la formación de TAGs que son depositados en el hepatocito. Sin embargo, el hígado tiene una capacidad limitada para la acumulación de TAGs, el cual favorece a la muerte celular debido a los depósitos de lípidos ${ }^{59}$.

Estudios experimentales indican que el exceso de $A G$ s condiciona altas tasas de $\beta$-oxidación con producción de EROs, como el $\mathrm{O}_{2}$, radical superóxido y peróxido de hidrógeno $\left(\mathrm{H}_{2} \mathrm{O}_{2}\right)$ a nivel de la cadena respiratoria de la mitocondria asociada a la inducción de necrosis celular.

A nivel hepático, los pacientes obesos presentan:

- Disminución de antioxidantes (menor actividad de superóxido dismutasa y contenido de glutatión).

- Aumento en la actividad pro-oxidante (mayor lipo peroxidacion, contenido de hidroperóxido y oxidación de proteínas).

- Activación de células de Kupffer (mayor producción del radical superóxido y tasa lipo per oxidativa) que implica fibrogénesis.

- Disminución de la capacidad antioxidante del plasma ${ }^{13}$. 
Actualmente el tratamiento de HGNA se reduce a pérdida paulatina de peso, control de la glicemia y abstinencia alcohólica. Sin embargo, estudios actuales han demostrado beneficios de ciertas sustancias antioxidantes como el probucol, que disminuye a dosis bajas los niveles de transaminasas en pacientes con EHNA. Además, estudios recientes han demostrado que en el EHNA existe activación e hiperplasia de células de Kupffer y células estrelladas, las cuales, permiten la expresión de genes fibrogénicos, proceso que puede ser inhibido por la $\alpha$-tocoferol ${ }^{60}$.

En el estudio se observó que en pacientes con obesidad grado I con índice de masa corporal mayor de 29, presentaban esteatosis hepática, evidenciada por exámenes de laboratorio, ultrasonografía y el QRS.

Dos pacientes presentaron un score de fibrosis EHNA negativo. Los cuales implicaban presencia de esteatosis moderada, con menor estrés oxidativo, ausencia de necrosis y de hepatitis crónica. Sin embargo uno de ellos PP002 presentó fibrosis indeterminada (fibrosis grado 1-2). La esteatosis simple es la etapa más precoz y prevalente y se considera que sensibiliza al hígado de agentes injuriantes nocivos, promoviendo la progresión de la enfermedad hacia esteatohepatitis y fibrosis.

El estrés oxidativo puede ser la causa y progresión de la enfermedad, dado que los EROs pueden dañar en forma directa alterando biomoléculas esenciales, con la siguiente perdida de su función, o bien indirectamente al activar las células de Kupffer y factores de transcripción redox-sensibles (NF-Kb, AP-1), lo que a su vez incrementan la síntesis de mediadores citotóxicos y fibrogénicos ${ }^{13}$.

Existe evidencia que sugiere la participación del estrés oxidativo crónico en la progresión del HGNA hacia esteatohepatitis y fibrosis hepática. En estos pacientes se ha demostrado un aumento de contenido de citocromo P450 y marcadores de peroxidación de lípidos ${ }^{59,60}$.
El aumento intracelular de ácidos grasos es por sí mismo un factor que induce la actividad del citocromo P450, 2E-1 y P450 4 A 12, que genera EROs con potencial cito toxico. Los productos de esta oxidación son 4-hidroxinonenal (4HNE) y el malondialdehido (MDA), los cuales son muy tóxicos, produciendo daño asociado con la Interleucina 8(IL-8), estimulando la quimio taxis de neutrófilos que a su vez favorece la producción del factor de crecimiento beta (TFG- $\beta$ ), el cual transforma e induce las células estrelladas (almacenadoras de vitamina A) a un estado mío-fibroblástico, produciendo fibrosis. El stress oxidativo induce además una depleción de factores protectores intracelulares como el glutatión, empeorando el daño a nivel del hepatocito ${ }^{13}$.

En este estudio, los pacientes obesos con esteatosis en grado moderado y tratados con antioxidantes por seis meses, teóricamente normalizaron su metabolismo aeróbico hepático. Es decir lograron una producción basal de especies reactivas de oxigeno (EROs), que fueron anulados en proporción por los mecanismos antioxidantes, debido a que la esteatosis disminuyó y siendo verificado por pruebas bioquímicas y de imagen e incluso el paciente PP002 con puntuación de fibrosis indeterminada mejoró en el periodo de un semestre.

Finalmente, se observó que los tres casos normalizaron sus enzimas ALT y AST, y en igual forma el monitoreo con el QRS. Uno de los casos (PP002) normalizó al quinto mes de tratamiento $\mathrm{y}$ dos normalizaron en el sexto mes (CA001 VH003). Sin embargo, en la ultrasonografía hepática, dos de ellos no mostraron esteatosis. Y al paciente PP002 se le diagnosticó esteatosis leve.

\section{CONCLUSIONES}

Al finalizar la terapia de antioxidantes se observa en los pacientes una disminución de los parámetros clínicos, bioquímicos, ultrasonográficos y el QRS de esteatosis hepática. Dos de ellos normalizaron por completo. 
- Probablemente el tratamiento de pacientes que presenta hígado graso no alcohólica (HGNA) con antioxidantes es efectivo, siempre y cuando se prolongue por un periodo mínimo de seis meses.

- Posiblemente la terapia antioxidante debe de incluir un número importante de compuestos, incluyendo los mecanismos de prevención, reparación y eliminación de radicales libres que permitan elevar los niveles deficientes de antioxidantes en cada paciente, en especial aquellos que favorezcan la síntesis endógena de glutatión, debido a que existe suficiente evidencia científica que avala su función como hepatoprotector y está descrita su sinergia con el ácido alfa lipoico.

- El Sistema Cuántico Bioeléctrico (QRS) es un método que presentó verificación de los hallazgos en forma satisfactoria con pruebas bioquímicas de laboratorio; evidencia clínica de obesidad (IMC) y cambios en la ultrasonografía hepática. Este método se basa en principios básicos de investigación científica que incluye: replicabilidad, racionalidad, principio teórico y principio de evidencia.

\section{AGRADECIMIENTOS}

Dra. Corina Esmeralda Arévalo de Acevedo. Médico Internista del Hospital General del Seguro Social de El Salvador, por su dedicación y tiempo en la revisión de este documento.

\section{REFERENCIAS BIBLIOGRÁFICAS}

1. Organización Mundial de la Salud (OMS) Obesidad y sobrepeso. Nota descriptiva $N^{\circ} 311$ enero 2015.

2. Browning JD, Szczepaniak LS, Dobbins R, Núremberg P, Horton JD, Cohen JC, Grundy SM, Hobbs HH. La prevalencia de la esteatosis hepática en una población urbana en los Estados Unidos: el impacto de la etnicidad. Hepatología. 2004; 40: 1387-95.

3. Ventilador J, Zhu J, Li X, Chen L, Li L, Dai M, Li F, Chen S. Prevalencia y factores de riesgo de hígado graso en una población general de Shanghái, China. J Hepatol. 2005; 43: 508-14.
4. Alberto Videla L, Obregón AM, Pettinelli P. Patología de Hígado Graso no-alcohólico (HGNA) asociada a obesidad: mecanismos patogénicos. Medwave 2011; 11(07): 5068.

5. López A L. Fernando C, Zelmira Lazarova, Bañuelos RV Sánchez SH. Antioxidantes, un paradigma en el tratamiento de Enfermedades. Revista Anacem 2012; Vol.6 (1): 12-14.

6. Coronado H M, Vega y León S, Gutiérrez R, Vásquez M, Radilla C, Antioxidantes: Perspectiva actual para la salud humana. Rev Chil Nutri 2015; 42 (2): 206211.

7. Brasier AR (2006) The NF-Kb Regulatory netwoek. Cardiovasc. Toxicol. 2006; 6(2): 111-30.

8. Núñez Sellés A. Terapia antioxidante, estrés oxidativo y productos antioxidantes: retos y oportunidades. Rev.Cubana Salud Publica Vol. 37(suple 5) 2011.

9. Merat S, Malekzadeh R, y Cols. Probucol in the treatment of no alcoholic steatohepatitis: an open labeled study. J Clin Gastroenterol. 2003; v (3): 266-268.

10. Phung N, Farrell GC, Robertson, G, George L. Vitamin E but not Glutathione precursors inhibit hepatic fibrosis in experimental, NASH exhibiting oxidative stress and mitochondrial abnormalities. Hepatology 2001; 34: 36.

11. Shenkin A. The key of micronutrients. Clin Nutr 2016. (1): 1-13.

12. Stanner SA, Hughes J, Kelly CN, Buttriss J. A review of the epidemiological evidence for the antioxidant hypothesis. Public Health Nutr 2014.7 (3): 407-22.

13. Martin D V, González J J, García B L, Moreno O R. Etiopatogenia, diagnóstico y tratamiento de la enfermedad de hígado graso no alcohólico. 2013. Rev Esp Enferm Diag. Madrid; 105(7): 409-420.

14. Woodside G, J.McCall D, McGartland C, Young I. La ingesta de carbohidratos, la obesidad, el síndrome metabólico y el riesgo de cáncer Proc Nut Soc 2015; 64(4): 543-53.

15. Tilh H, Diehl AM. Cytokines in alcoholic y no alcoholic steatohepatitis. New Engl J Med 2000; 343: 1467-76.

16. Poniachik J. Esteatohepatitis alcohólica y no alcohólica. Gastrolatinoam 2002; 13; (12): 55-8. 
17. Bedogni G, Miglioli L, Masutti F, Tiribelli C, Marchesini G, Bellentani S. Prevalencia y factores de riesgo de enfermedad del hígado graso no alcohólico: la nutrición Dionysos y el estudio del hígado. Hepatología. 2005; 42: 44-52.

18. Rodrigo A, Carrasco L, Rodrigo. L. Castillo P. Patricio. A. Huerta B. Lilian Thieleman. La enfermedad de hígado graso no alcohólica y su asociación con obesidad y estrés oxidativo hepático. Medunab 2013; 6(16): 15-20.

19. Foruny Olcina JR, Albillos Martínez A. Actitud diagnóstica ante una hipertransaminasemia. Jano 2006; 12(1): 622 .

20. Csendes P, Paulinelli P, Busel D M, Venturelli V, Rodríguez S. Hígado graso: Ultrasonido correlación anatomopatologica. Revista Chilena de Radiología 2004; (10); 2; 51-52.

21. Nomura H, Kashiwagi S, Hayashi J, Kajiyama W, Tani S, Goto M. Prevalencia de hígado graso en una población general de Okinawa, Japón. Japonés J Med. 1988; 27: 142-9.

22. González, Pérez B, Salas Flores R. Esteatosis hepática en niños obesos Revista de Endocrinología y Nutrición 2008; 16(2): 74-82

23. Analizador cuántico de resonancia magnética. Manual del usuario.

24. Marceau P, Biron S, Hould ES, et al. Liver Pathology and the metabolic syndrome $\mathrm{X}$ in severe obesity. J Clin Endocrinol Metab 1999; 84: 15113-7.17

25. Nakao K, Nakata K, Ohtsubo N, et al. Association between nonalcoholic fatty liver, markers of obesity, and serum leptin level in young adults. Am J Gastroenterol 2002; 97: 1796-801.

26. Castera L, Vilgrain V, Angulo P. Evaluación no invasiva de hígado graso no alcohólico. Nat Rev Gastroenterol Hepatol. 2013; 10: 666-675.

27. Angulo $\mathrm{P}$, Keach JC, Batts KP, Lindor KD. Independent predictors of liver fibrosis in patients with nonalcoholic Steato-hepatitis. Hepatology. 1999; 30: 1356-62

28. Méndez Sánchez, Chávez N C, Uribe M. Hígado graso no alcohólico. Nuevos conceptos. Rev Invest Clin 2004; Vol. 56(1): 72-82.
29. Matteoni CA, et al. Nonalcoholic fatty liver disease: a spectrum of clinical and pathological severity. Gastroenterology 1999; 116:1413-9.

30. Gutiérrez V. Daño oxidativo radicales libres y antioxidantes. Rev. Cubana Med. Milit 2002; 31(2): 126-33.

31. Carrillo Espera R, Muciño Bermejo B. Conceptos actuales de hígado graso y esteatohepatitis no alcohólica. Revista de Gastroenterología 2010; 75(2): 143-8

32. Salem G.I., Acevedo J. A. Beneficios de antioxidantes en el Hígado Graso No Alcohólico. San Salvador: Editorial UEES; Archivo de Departamento de Patología de la Universidad Evangélica de El Salvador de $2014-2016$.

33. Cisneros Prego E, La glutatión reductasa y su importancia Biomédica. Rev Cubana Invest Bioméd 1995; 14(1)95.

34. Sarrasague M, Barrado D, Zubillaga M, Hager A, De Paoli T, Boccio J. Conceptos actuales del metabolismo del glutatión. Utilización de los isotopos estables para la evaluación de su homeostasis. Acta Bioquim Clin Latinoam 2006; 40 (1): 45-51.

35. Martínez S J, Torres P V, Juárez M A. El glutatión y su asociación con las enfermedades neurodegenerativas, la esquizofrenia, el envejecimiento y la isquemia cerebral. REB 2011; 30 (2): 56-67.

36. Ying Chang et al, Effect of Cordyceps Militaris supplementation on sperm production, sperm motility and hormones in Sprague-Dawley rats. Am J. Chin. Med. 2008; 36(5): 849-59.

37. Constant J. Alcohol, isquemic heart disease, and French paradox. Clin Cardiol 1997; 20(5): 4020-4.

38. Sharma OP. Antioxidant activity of curcumin and related compounds. Biochem Pharmacol 1976; 25: 1811-1812.

39. Toda S, Miyase T, ArichiH, TanizawaH, Takino Y. Natural antioxidants IR Antioxidative components isolated from rhizome of Curcuma Longa L. Chem Pharm Bull, 1985; 33: 1725-1728.

40. Kaul S, Krishnakantha TP. Influence of retinol deficiency an curcumin/turmeric feeding on tissue microsomal membrane lipid per oxidation and fatty acids in rats. Mol Cell Bioquim 1997; 175(1-2): 43-48. 
41. Began G, Sudharshan E, Appu -Rao AG .Inhibition of lipoxygenase 1 by phosphatidylcholines micelles-bound curcumin. Lipids 1998; 33 (12): 12231228 .

42. Venkatesan N. Curcumin attenuation of acute Adriamycin myocardial toxicity in rats. J Pharmacol, 1998; 124: 425-427.

43. Osawa T, Sugiyama Y, Inayoshi M, Kawakishi S. Antioxidative activity of tetrahydrocurcuminoids. Biosci Biotechnol Biochem, 1995; 59(9): 1609-1612.

44. Zhao BL,LiXJ,He RG,Cheng SJ,Xin WJ, Scavenging effects of extracts of green tea and natural antioxidants on active oxygen radicals. Cell Biopsy's, 1995; 14: 175-185.

45. Priyadarsini KI. Free radical reactions of Curcumin in membrane models. Free Radic Biol Med, 1997; 23(6): 838-843.

46. Reddy AC, Lockesh, BR. Studies on the inhibitory effects of curcumin and eugenol on the formations of reactive oxygen species and the oxidation of ferrous iron. MOLL Cell Biochem, 1991; 137: 1-8.

47. Unnkrishnan MK, Rao MNA. Curcumin inhibits nitrite-induced methemoglobin formation. FEBS Let. 1997; 301: 195-196.

48. Unnkrishnan MK, Rao MNA. Curcumin inhibits nitrogen dioxide oxidation of hemoglobin. Mol Cell Biochem, 1995; 146: 35-37.

49. Hidalgo M, López M, Fernández E, Cabrera E, Goecke H, Ruz J. Efecto antioxidantes de un extracto de semilla de uva en pacientes diabéticos tipo 2 . Revista de fitoterapia 2006; 6(2): 137-142.

50. Schwartz K, Foltz CM. Selenium is an integral part of factor 3 against dietary necrotic liver degeneration. J Amer Chem Soc. 1957; 79: 3292-3293.

51. Flohé L. The glutathione peroxidase reaction: molecular basis of the antioxidant function of selenium in mammals. Curr Top Cell Regul.1985; 27: $473-478$.
52. Shibata Y, Morita M, Fuwa K. Selenium and arsenic in biology: Their chemical forms and biological functions. Adv Biophy. 1992; 28: 31-80.

53. Durand M, Mach N. El ácido alfa lipoico y su poder antioxidante frente al cáncer y las patologías de sensibilización central. Nutr Hosp. 2013; 28(4): 1031-1038.

54. Rambaldi A, Jacobs BP, Gluud C. Cardo mariano para la hepatopatía alcohólica y/o la hepatitis viral B o C. La Biblioteca Cochrane Plus, 2008; 4(5.); 17459990.

55. Fahey JW, Zhang Coles de Talala y. Broccoli sprouts: An exceptionally rich source of inducers of enzymes that protect against chemical carcinogens. Proc Natl Acad. 1997; 94(19): 10367-10372.

56. Calderon-Oliver M. Quiñones P M. Efectos beneficiosos del Aloe en la salud. Rev. Especializada en Ciencias de la Salud 14(2): 53-73, 2011.

57. Ui-Hyun Park, Hong-Suk Jeong, Eun-Young Jo, Taesun Park, Seung Kew Yoon,Eun-Joo Kim, Ji-Cheon Jeong, and Soo-Jong Um Piperine, a Component of Black Pepper, Inhibits Adipogenesis by Antagonizing PPAR $\gamma$ Activity in 3T3-L1 Cells.». Journal of Agricultural and Food Chemistry" 60 (15): 3853-3860.

58. Almarza J. Hígado graso no alcohólico: ¿un componente inflamatorio del síndrome metabólico? Perspect Nutr Humana. 2010; 12: 163-175.

59. Foruny Olcina JR, Albillos Martínez A. Actitud diagnóstica ante una hipertransaminasemia. Jano 2006; 12(1): 622 .

60. Carvajal Carvajal C. Tejido adiposo, obesidad, insulina resistencia. Med. Leg. Costa Rica 2015. vol. 32(2). 